\title{
Silicon photonic Mach Zehnder modulators for next-generation short- reach optical communication networks (Invited)
}

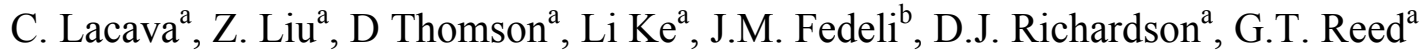 \\ and P. Petropoulos ${ }^{\mathrm{a}}$ \\ ${ }^{a}$ Optoelectronics Research Centre, University of Southampton, Highfield Campus, \\ Southampton SO17 1BJ, United Kingdom \\ ${ }^{\mathrm{b}}$ CEA LETI, Minatec Campus, Grenoble 38054, France
}

\begin{abstract}
Communication traffic grows relentlessly in today's networks, and with ever more machines connected to the network, this trend is set to continue for the foreseeable future. It is widely accepted that increasingly faster communications are required at the point of the end users, and consequently optical transmission plays a progressively greater role even in short- and medium-reach networks. Silicon photonic technologies are becoming increasingly attractive for such networks, due to their potential for low cost, energetically efficient, high-speed optical components. A representative example is the silicon-based optical modulator, which has been actively studied. Researchers have demonstrated silicon modulators in different types of structures, such as ring resonators or slow light based devices. These approaches have shown remarkably good performance in terms of modulation efficiency, however their operation could be severely affected by temperature drifts or fabrication errors. Mach-Zehnder modulators (MZM), on the other hand, show good performance and resilience to different environmental conditions. In this paper we present a CMOS-compatible compact silicon MZM. We study the application of the modulator to short-reach interconnects by realizing data modulation using some relevant advanced modulation formats, such as 4-level Pulse Amplitude Modulation (PAM-4) and Discrete MultiTone (DMT) modulation and compare the performance of the different systems in transmission.
\end{abstract}

Keywords: silicon photonics, Mach-Zehnder modulator, high speed link, short reach optical connections

\section{INTRODUCTION}

Fibre optic-based DWDM (Dense Wavelength Division Multiplexing) communication systems are widely used in longdistance communication links for large bandwidth optical transmission connections. In recent years, the continuous growth of data transmitted in communication networks, the need to connect a large number of smart devices to the networks and the advent of large data cloud systems require larger and faster data-centers that should have the capability of rapidly handling a large amount of data whilst keeping a low power consumption. Substantial advances in the area of electronic microprocessors have given rise to outstanding levels of integration. However, copper-based interconnects are starting to show their limitations in terms of bandwidth, heat dissipation and crosstalk. Several solutions have been proposed to overcome these limitations such as the use of thicker metal pits or of exotic materials with higher dielectric constants. Nevertheless, all of these potential alternatives amount to rather expensive solutions making indeed, the possibility to apply silicon photonics-based optical communication technologies in rack-to-rack, board-to-board or even intra-chip interconnects a very attractive option [1], [2].

Many high speed optical components that have the potential to be integrated on the same monolithic chip yielding compact, low cost, CMOS compatible optical transceivers have already been demonstrated, such as waveguiding components [3], high speed optical modulators [4]-[6] and high speed detectors [7], [8].

When considering short-link connections $(<1 \mathrm{~km})$ one of the most critical components on the transmitter side is the optical modulator. Different device schemes have been widely studied in the last decade including Mach-Zehnder interferometer (MZI) based modulators [9]-[12], ring resonator devices [13] and electro-absorption modulators (EAM) [14]. Carrier depletion MZI modulators have shown very good performance in terms of offered bandwidth [4] and resilience to various external conditions. Moreover, in contrast to ring resonator-based modulators, MZI-based devices 
are wavelength transparent, which allows flexible and reconfigurable operation. $50 \mathrm{~Gb} / \mathrm{s}$ operation has already been demonstrated using On Off Keying (OOK) modulation [15], showing a $3 \mathrm{~dB}$ extinction ratio that could allow error-free operation in a short reach optical connection. Further design improvements could lead to even higher bit-rate operation with improved signal to noise ratio performance [16]. The analysis presented in [16] clearly highlights that the bottleneck in increasing further the operational bit-rates is not represented by the optical technology but rather the driving integrated electronics that needs to exhibit a very high bandwidth ( $>50 \mathrm{GHz}$ to achieve $100 \mathrm{~Gb} / \mathrm{s}$ operation) combined with, possibly low-cost fabrication.

In order to achieve even higher operation speeds (e.g. $400 \mathrm{Gbit} / \mathrm{s}$ is a useful benchmark), high-order modulation formats are needed. Recently, modulation formats, such as $n$-level Pulse Amplitude Modulation (PAM- $n$ ) [17] and Discrete Multitone Transmission (DMT) [18] are considered as a potential route for achieving this goal. First demonstrations that have examined the compatibility of these formats with MZI silicon modulators has been reported [10], [19]. In this paper we present experiments that study the application of both PAM-4 and DMT modulation techniques in short reach links $(<10 \mathrm{~km})$ using a silicon MZI modulator, and compare the performance of these modulation techniques in different transmission schemes.

\section{SILICON OPTICAL MODULATOR}

The silicon optical modulator structure consists of a MZI with a phase modulator in each arm. Low loss 1x2 Multimode interference structures are used to split and recombine the light from the two waveguide arms [20]. The phase modulators are based on the plasma dispersion effect [21] which relates changes in electron and hole concentration in the waveguide to changes in refractive index (and absorption). The density of electrons and holes in interaction with the propagating light is electrically controlled using a pn diode structure at the waveguide. When a reverse bias is applied to the diode, carriers are depleted from the junction, therefore causing a change in the refractive index. A cross-section of the phase modulator structure is shown in Fig.1.

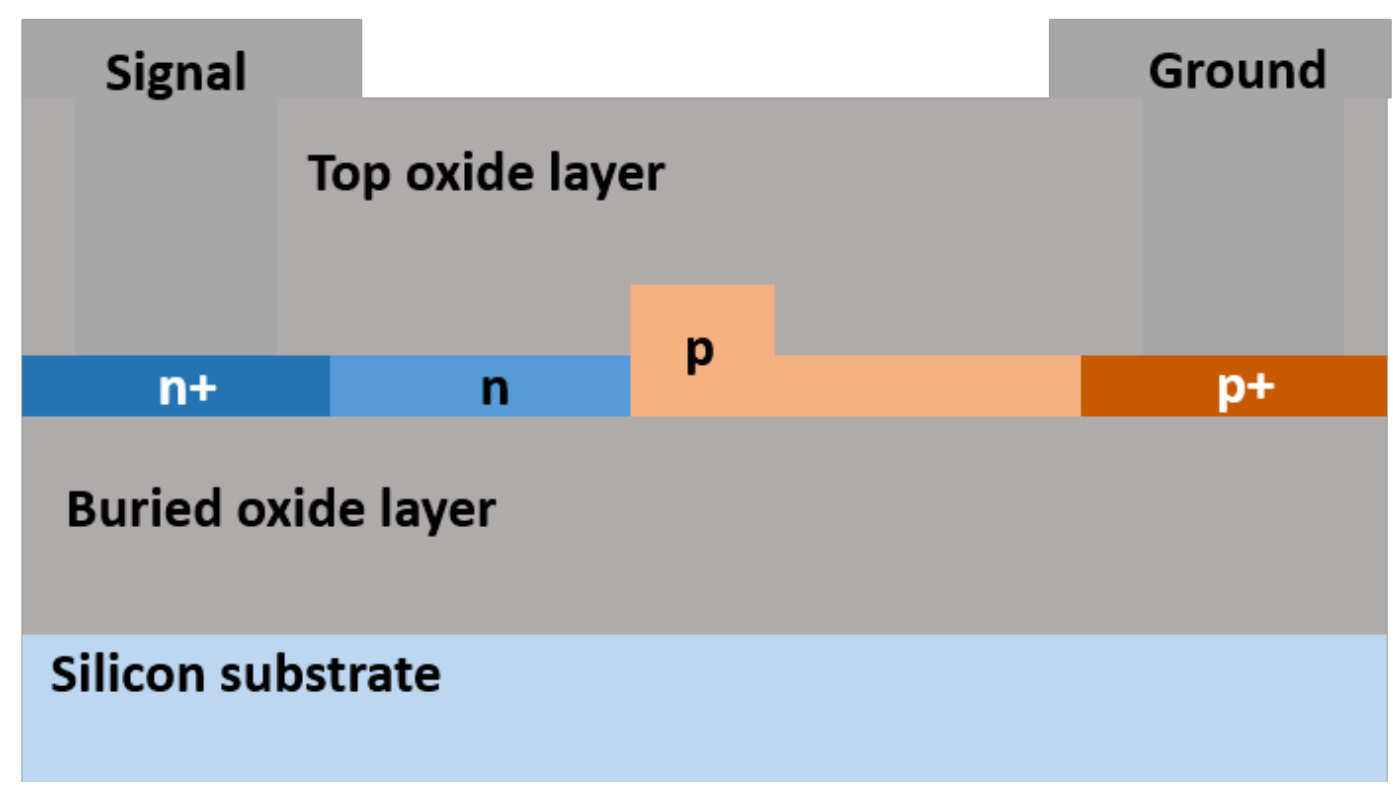

Figure 1 - Cross-sectional diagram of a silicon phase modulator.

The waveguide is of dimensions $220 \mathrm{~nm}$ height, $400 \mathrm{~nm}$ width and $100 \mathrm{~nm}$ slab height, which allow only single mode propagation. The waveguide sits on a $2 \mu \mathrm{m}$ buried silicon dioxide layer and has a $1 \mu \mathrm{m}$ thick silicon dioxide upper cladding layer. The rib section and slab to one side is doped p-type and the slab to the other side is doped n-type, forming a junction coinciding with one edge of the waveguide. The diode is configured in this way so that it can be formed using a self-aligned process as reported previously [16]. This results in a device that is resilient to typical fabrication variations. 
In order for the depletion to extend mostly into the rib section during reverse bias application, the doping concentration of the p-type region $\left(3 \cdot 10^{17}\right)$ is made much lower than the n-type region $\left(1.5 \cdot 10^{18}\right)$, meaning that efficient operation can be achieved. High doped $\mathrm{p}+$ and $\mathrm{n}+$ regions are positioned in the slab regions at distances of $450 \mathrm{~nm}$ and $500 \mathrm{~nm}$ from the waveguide edge respectively to allow ohmic contacts to be formed with the device. Coplanar waveguide electrodes designed to have a characteristic impedance of $50 \mathrm{Ohms}$ are formed on the devices to allow the phase modulators to be driven at high speed. Figure 2 shows the final device arrangement. The dashed line represents the optical waveguide circuitry while the continuous line represents the electrical part. The phase-shifter length was equal to $1 \mathrm{~mm}$.

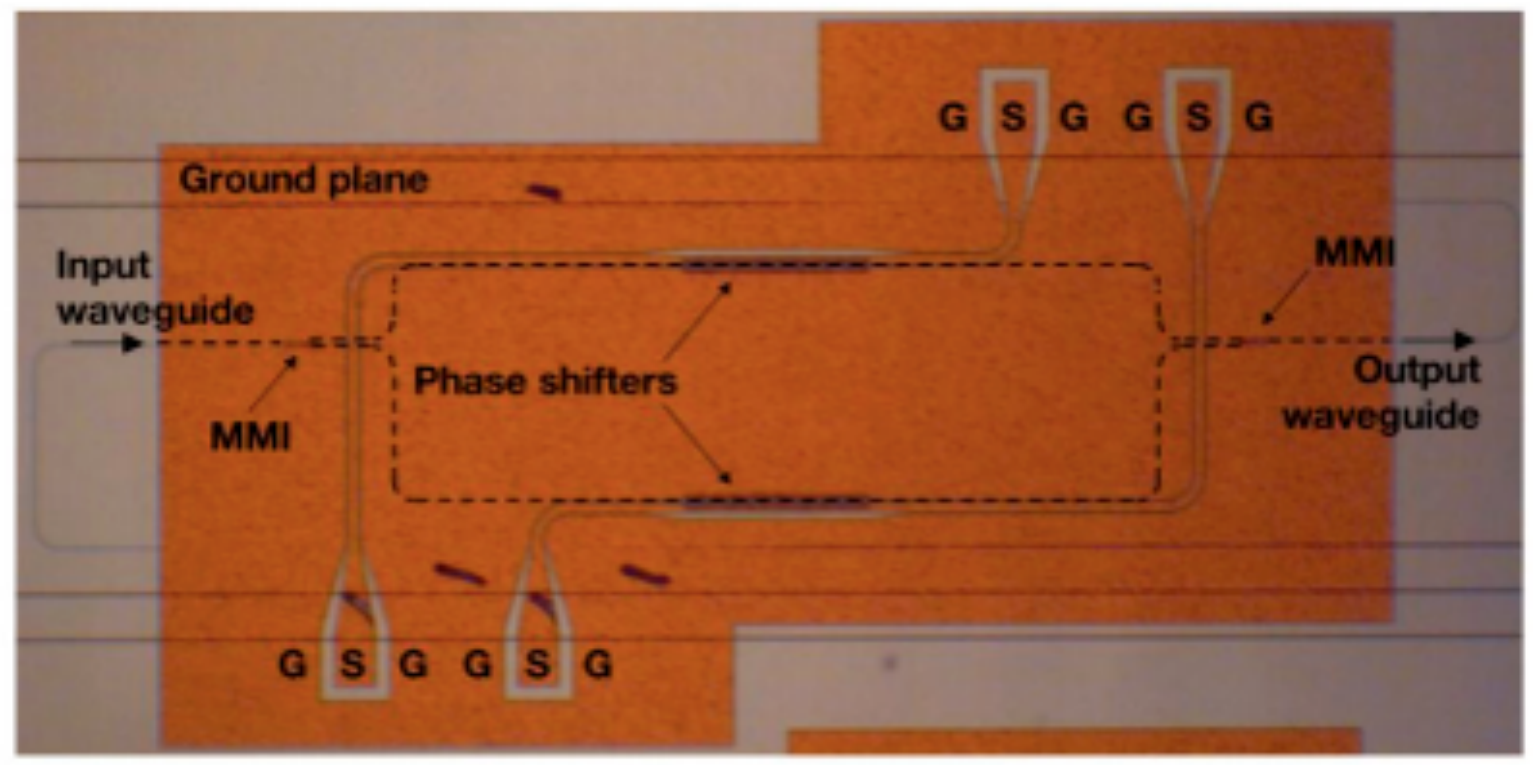

Figure 2: Top view of MZI modulator device. The phase shift length was $1 \mathrm{~mm}$.

\section{DICRETE MULTITONE TRANSMISSION MODULATION}

DMT is an OFDM-based modulation scheme that transmits information by modulating only the real part of the signal. Since no information is carried by the signal phase, an optical intensity modulator can be used for data encoding on the optical carrier. Figure 3 shows the experimental set-up that was used to carry out the designed measurements. The DMT waveform samples were generated offline based on a PRBS of length $2^{18}-1$ and an inverse Fourier transform with a size of 1024. The modulation format for each sub-carrier was assigned by a bit loading algorithm. A 16-sample cyclic prefix was placed both before and after each symbol. The generated signal was transferred into the electrical domain by means of an Arbitrary Waveform Generator (AWG) operated at $24 \mathrm{GS} / \mathrm{s}$. A $15 \mathrm{GHz}$ RF amplifier was used to boost the electrical signal; a multiprobe system was then employed to drive the silicon MZI modulator. At the receiver, a $12.5 \mathrm{GHz}$ photodiode was placed, then the electrical signal was sent into an $80 \mathrm{GS} / \mathrm{s}$ real-time scope that recorded and stored the signal samples for subsequent offline signal processing. By employing our receiver off-line we were able to measure the obtained channel capacity at a specific bit-error ratio (BER) level. Results showed that this system supports a maximum capacity of $22 \mathrm{~Gb} / \mathrm{s}$ (for a BER $=4 \cdot 10^{-3}$, i.e. below the FEC threshold) when a $10 \mathrm{~km}$ single-mode fiber (SMF) was placed between the MZI modulator and the receiver. When the transmission fiber was removed, the capacity was increased to a maximum value of $25 \mathrm{~Gb} / \mathrm{s}$ at the same BER level. It is worth noting that the main limitations in the current experiment were represented by the bandwidth of the available electronic equipment (RF amplifier and AWG). Use of faster driving electronics would allow us to achieve even higher transmission rates and/or better signal quality. The allocated DMT spectrum and the bit allocation scheme are shown in Fig. 4. The figure shows that a maximum of $5 \mathrm{bit} / \mathrm{carrier}$ were successfully allocated in the low-frequency region. 




Figure 3 - DMT modulation experimental set-up
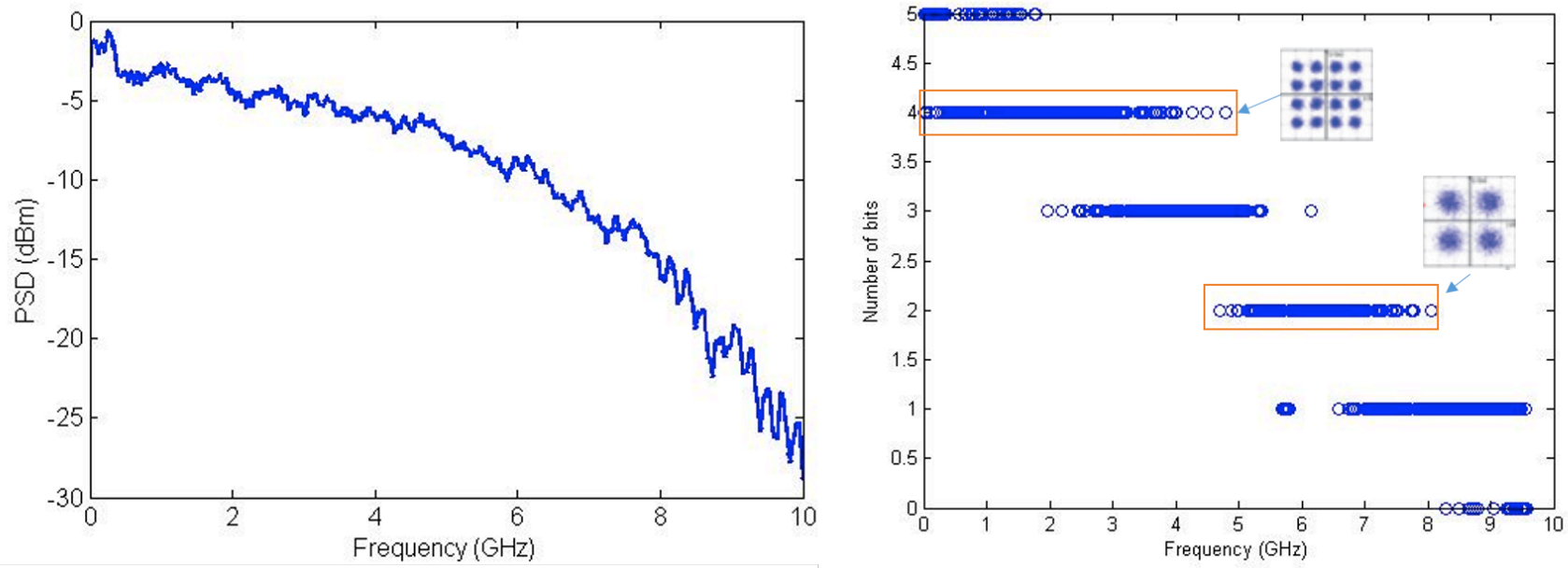

Figure 4 - Results recorded during DMT transmission; electrical spectrum (left) and bit allocation per subcarrier (right) after $10 \mathrm{~km}$ SMF transmission.

\section{PAM-4 MODULATION EXPERIMENT}

In order to test the performance of our system when employing PAM-4 modulation, we used a similar experimental setup as before, as shown in Fig.5. A 10 Gbaud test signal was directly generated by means of a 24 GS/s AWG. The electrical signal was then amplified by means of a $15 \mathrm{GHz}$ RF amplifier, then converted into the optical domain by means of the silicon MZI modulator. A $10 \mathrm{~km}$ SMF was placed after the modulator to test the signal performance when short reach propagation is considered. After fibre propagation the signal was detected using a $12.5 \mathrm{GHz}$ bandwidth 
photodiode which connected to an $80 \mathrm{GS} / \mathrm{s}$ analog-to-digital convertor (ADC). An offline routine was used to measure the signal performance and evaluate the BER of the received signal. In the inset to Fig. 5 we show an example of a recorded waveform when a 10 Gbaud signal $(20 \mathrm{Gbit} / \mathrm{s})$ was sent inside the MZI optical modulator. A minimum BER of $3 \cdot 10^{-3}$ was achieved at this operating speed.

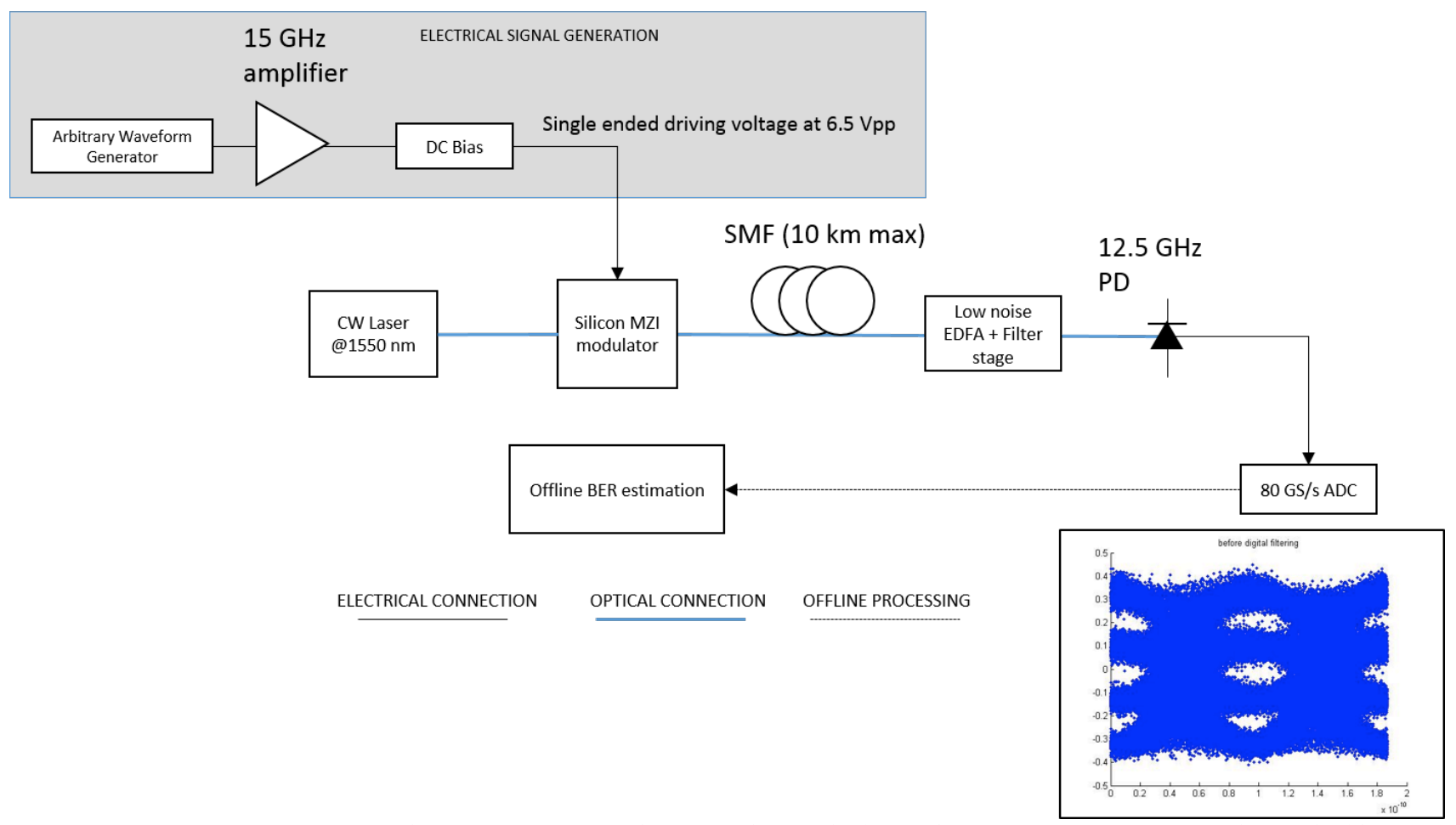

Figure 5: PAM-4 experimental set-up; inset: eye diagram obtained after 10km transmission in a single-mode fibre.

\section{CONCLUSION}

In this work we have presented results of both DMT and PAM-4 transmission using a silicon photonics MZI intensity modulator. DMT modulation has shown better performance in terms of overall system capacity, providing an error-free (considering use of FEC) $22 \mathrm{Gbit} / \mathrm{s}$ capacity after $10 \mathrm{~km}$ single mode fibre propagation. Use of PAM-4 modulation allowed similar performance to be achieved when the operating speed was $20 \mathrm{Gbit} / \mathrm{s}$. Further experimental improvements are anticipated by improving the electrical signal bandwidth, which would allow a higher transmission capacity to be demonstrated. These results show that our silicon MZI modulator is suitable for short-reach optical links and both PAM4 and DMT modulation techniques could be considered as viable alternatives for such a purpose.

\section{ACKNOLEDGMENTS}

This work has been supported by EPSRC, UK through the Silicon Photonics for Future Systems Programme Grant. G.T. Reed is a Royal Society Wolfson Research Merit Award holder. He is grateful to the Wolfson Foundation and the Royal Society for funding of the award.

\section{REFERENCES}

[1] B. Jalali and F. Sasan, "Silicon photonics," J. Light. Technol., vol. 24, no. 12, pp. 4600-4615, Dec. 2004.

[2] R. G.T, "An Introduction to Silicon Photonics and a brief history of the field," Electron. Eng., 2011.

[3] G. T. Reed, Silicon Photonics: The state of the Art, First Ed. Chichester: John Wiley \& Sons, Inc., 2008 , p. 330. 
[4] G. T. Reed, G. Z. Mashanovich, F. Y. Gardes, M. Nedeljkovic, Y. Hu, D. J. Thomson, K. Li, P. R. Wilson, S.-W. Chen, and S. S. Hsu, "Recent breakthroughs in carrier depletion based silicon optical modulators," Nanophotonics, vol. 3, no. 4-5, pp. 229-245, Jan. 2014.

[5] K. Li, D. J. Thomson, S. Liu, P. Wilson, and G. T. Reed, “A 30 Gb / s CMOS driver integrated with silicon photonics MZM."

[6] M. Gould, T. Baehr-Jones, R. Ding, S. Huang, J. Luo, A. K.-Y. Jen, J.-M. Fedeli, M. Fournier, and M. Hochberg, "Silicon-polymer hybrid slot waveguide ring-resonator modulator.," Opt. Express, vol. 19, no. 5, pp. 3952-61, Feb. 2011.

[7] E. Temporiti, G. Minoia, M. Repossi, D. Baldi, A. Ghilioni, and F. Svelto, “A 3D-Integrated 25Gbps Silicon Photonics Receiver in PIC25G and 65nm CMOS technologies,” Essderc Esscirc, pp. 131-134, 2014.

[8] S. Lishke, D. Knoll, C. Mai, A. Peczek, K. Voigt, E. Krune, K. Petermann, L. Zimmermann, and A. Mai, "Highbandwidth waveguide-coupled Ge pin photodiode with high $\mathrm{C}$ and L band responsivity," Gr. IV Photonics (GFP), 2015 12th IEEE Int. Conf., 2015.

[9] D. J. Thomson, F. Y. Gardes, Y. Hu, G. Mashanovich, M. Fournier, P. Grosse, J.-M. Fedeli, and G. T. Reed, "High contrast 40Gbit/s optical modulation in silicon.," Opt. Express, vol. 19, no. 12, pp. 11507-11516, 2011.

[10] K. Xu, L. Yang, and J. Sung, "Compatibility of silicon Mach-zehnder modulators for advanced modulation formats," Light. ..., vol. 31, no. 15, pp. 2550-2554, 2013.

[11] S. Akiyama, T. Baba, M. Imai, T. Akagawa, M. Takahashi, N. Hirayama, H. Takahashi, Y. Noguchi, H. Okayama, T. Horikawa, and T. Usuki, "125-Gb/s operation with 029-V·cm V_ $\pi \mathrm{L}$ using silicon Mach-Zehnder modulator based-on forward-biased pin diode," Opt. Express, vol. 20, no. 3, p. 2911, 2012.

[12] C. Lacava, M. J. Strain, P. Minzioni, I. Cristiani, and M. Sorel, "Integrated nonlinear Mach Zehnder for 40 Gbit/s all-optical switching," Opt. Express, vol. 21, no. 18, p. 21587, Sep. 2013.

[13] W. Bogaerts, P. De Heyn, T. Van Vaerenbergh, K. De Vos, S. Kumar Selvaraja, T. Claes, P. Dumon, P. Bienstman, D. Van Thourhout, and R. Baets, "Silicon microring resonators," Laser Photon. Rev., vol. 6, no. 1, pp. 47-73, Jan. 2012

[14] Y. Tang, H.-W. Chen, S. Jain, J. D. Peters, U. Westergren, and J. E. Bowers, "50 Gb/s hybrid silicon travelingwave electroabsorption modulator.," Opt. Express, vol. 19, no. 7, pp. 5811-5816, 2011.

[15] D. J. Thomson, F. Y. Gardes, J.-M. Fedeli, S. Zlatanovic, Y. Hu, B. P. P. Kuo, E. Myslivets, N. Alic, S. Radic, G. Z. Mashanovich, and G. T. Reed, "50-Gb/s Silicon Optical Modulator," IEEE Photonics Technol. Lett., vol. 24, no. 4, pp. 234-236, 2012.

[16] D. Thomson, F. Gardes, S. Liu, H. Porte, L. Zimmermann, J. Fedeli, Y. Hu, M. Nedeljkovic, X. Yang, P. Petropoulos, and G. Mashanovich, "High Performance Mach Zehnder Based Silicon Optical Modulators," IEEE J. Sel. Top. Quantum Electron., vol. 19, no. 6, p. 3400510, 2013.

[17] K. Szczerba, P. Westbergh, M. Karlsson, P. a. Andrekson, and A. Larsson, "70 Gbps 4-PAM and 56 Gbps 8PAM Using an $850 \mathrm{~nm}$ VCSEL,” J. Light. Technol., vol. 33, no. 7, pp. 1395-1401, 2015.

[18] P. Dong, J. Lee, Y. Chen, L. L. Buhl, S. Chandrasekhar, J. H. Sinsky, and K. Kim, "Four-Channel 100-Gb/s per Channel Discrete Multi-Tone Modulation Using Silicon Photonic Integrated Circuits,” Opt. Fiber Commun. Conf. Post Deadline Pap., vol. 1, no. d, p. Th5B.4, 2015.

[19] Z. Liu, Y. Chen, Z. Li, B. Kelly, R. Phelan, J. O. Carroll, T. Bradley, J. P. Wooler, N. V Wheeler, A. M. Heidt, T.Richter, S. Member, C. Schubert, M. Becker, F. Poletti, M. N. Petrovich, S. Member, S. Alam, S. Member, and D. J. Richardson, "High-Capacity Directly Modulated Optical Transmitter for 2- $\mu \mathrm{m}$ Spectral Region," J. Light. Technol., vol. 33, no. 7, pp. 1373-1379, 2015.

[20] D. J. Thomson, Y. Hu, G. T. Reed, and J. M. Fedeli, "Low loss MMI couplers for high performance MZI modulators," IEEE Photonics Technol. Lett., vol. 22, no. 20, pp. 1485-1487, 2010.

[21] M. Nedeljkovic, R. Soref, and G. Z. Mashanovich, "Free-Carrier Electrorefraction and Electroabsorption Modulation Predictions for Silicon Over the 1-14 um infrared wavelength range," IEEE Photonics J., vol. 3, no. 6, pp. 1171-1180, 2011. 